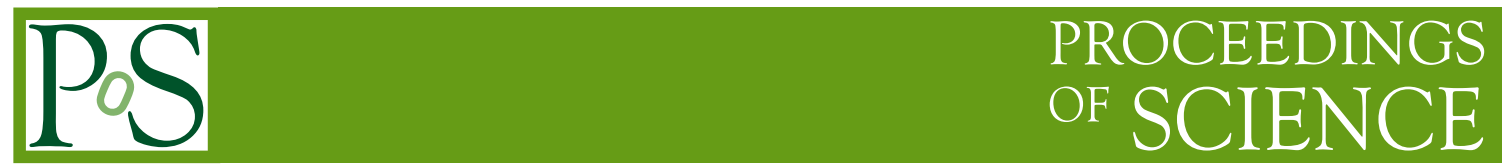

\title{
Overview of the $C P$ violation and mixing in the charm sector
}

\author{
Evelina Gersabeck* ${ }^{* \dagger}$ \\ Ruprecht-Karls-Universitaet, Heidelberg, Germany; now at the University of Manchester, \\ Manchester, $U K$ \\ E-mail: Evelina.Gersabeckecern.ch
}

An overview of the most recent experimental measurements in charm $C P$ violation and mixing parameters is presented here.

The 15th International Conference on Flavor Physics and CP Violation

5-9 June 2017

Prague, Czech Republic

* Speaker.

$\dagger$ on behalf of the LHCb collaboration with results from BELLE, BESIII, BaBar and CLEO-c legacy data 


\section{Introduction to mixing, $C P$ violation and the charm flavour factories}

The charm quark was discovered in 1974 [1], and for more that forty years particle physicists have been searching for matter-antimatter differences in the quark system. The mesons containing one charm quark are a superb laboratory to study the mixing and $C P$ violation phenomena in the uptype quark sector. Neutral charm mesons can oscillate, meaning they periodically change into their antimatter partner and vice versa. The mass eigenstates, with well defined masses $m_{1,2}$ and widths $\Gamma_{1,2}$, can be presented as a superposition of the flavour states: $\left|D_{1,2}\right\rangle=p\left|D^{0}\right\rangle \pm q\left|\bar{D}^{0}\right\rangle$ where $p$ and $q$ are complex numbers. The charm mixing observables are defined as $x=\frac{m_{2}-m_{1}}{\Gamma_{1}+\Gamma_{2}}$ and $y=\frac{\Gamma_{2}-\Gamma_{1}}{2\left(\Gamma_{1}+\Gamma_{2}\right)}$. By now the mixing of neutral charm mesons $D^{0}$ is firmly established. The no-mixing hypothesis has been excluded with a significance more than ten standard deviations form a single experiment [2]. However, there is no experimental evidence for $C P$ violation in the up-type quark sector. The $C P$ symmetry applies to processes invariant under the combined transformation of charge conjugation and parity. The $C P$ symmetry can be violated in several ways. If the quantity $\lambda_{f}=-\eta_{C P}\left|\frac{q}{p}\right|\left|\frac{\bar{A}_{\bar{f}}}{A_{f}}\right| e^{i \phi}$ is different than 1 , then the $C P$ symmetry is violated. Indirect $C P$ violation occurs when $\left|\frac{q}{p}\right| \neq 1$ or when the $C P$-violating relative phase $\phi \neq 0$. The direct $C P$ violation depends on the decay mode, and manifests itself when the amplitude for the decay of a particle, $A$, into a given final state, $f$ differs from the amplitude of the antiparticle decay, $\bar{A}$ into the charge-conjugate final state, $\bar{f}$, namely $\left|\bar{A}_{\bar{f}}\right| \neq 1$. The $C P$ violation within the Standard Model is contained in the imaginary part of the Cabibbo-Kobayashi-Maskawa (CKM) matrix [3] that governs the transitions between the different types of quarks. The violation of the $C P$ symmetry has been discovered in weak decays of $K$ and $B$ mesons containing down-type quarks. The amount of $C P$ violation in the up-type sector is expected to be rather small because of the CKM matrix structure - the imaginary part of the CKM element $V_{c d}$ is very small. However, it should be noted that the theoretically it is very difficult to predict precise quantities for the charm mesons because of their mass which does not allow effective theories to be used.

This brief overview includes the most recent experimental charm results of the two B factories, Belle [4] at the asymmetric-energy electron-positron collider, KEKB, and BaBar [5] at the PEP-II collider, results based on data taken with the CLEO-c detector [6] at the electron-positron collider CESR (using a legacy data set), results from the $\mathrm{LHCb}$ experiment $[7,8]$ at the proton-proton machine LHC, and from the chinese flavour factory BESIII [9] at the electron-positron collider, BEPCII.

There are several ways to identify the flavour of the neutral charm mesons. At the B factories and $\mathrm{LHCb}$, the prompt charm decays are tagged by the charge of the pion in the strong decay $D^{*+} \rightarrow D^{0} \pi^{+}$. At LHCb only, the charge of the muon in $B \rightarrow D \mu v_{\mu} X$ is used to define the flavour of $D^{0}$ originating from secondary charm decays. A special class of events called doubly-tagged (DT) events where the $D^{0}$ comes from decays $B \rightarrow D^{*+}\left(\rightarrow D^{0} \pi^{+}\right) \mu^{-} v_{\mu} X$ has a very low mistag probability. These events are exploited to obtain extra-clean samples of neutral charm meson decays, however the available DT data is significantly smaller than the prompt charm data. At LHCb, the singly-tagged secondary charm decays samples are about three times smaller than the ones of the prompt charm decays to the same final state. The prompt and secondary samples are statistically independent. The two types of charm events have a different decay-time acceptances, and to a 
large extent, the measurements with both types of events are complementary. In addition, there are different kinds of systematic uncertainties associated with both classes.

The flavour of charm mesons at CLEO-c and BESIII experiments is accessible through the quantum-entangled charm pairs of $D^{0} \bar{D}^{0}$ produced at the threshold of $\psi(3770)$ with no additional hadrons. The principle of quantum correlation states that by making a measurement on the particle in a given moment of time we find out information about the antiparticle state at that point of time as well. By looking at the opposite side of the event, decaying to either a flavour specific decay, or a $C P$ eigenstate, conclusions can be made about the signal decay.

\section{Mixing and $C P$ violation}

\subsection{Mixing and $C P$ violation in $D^{0} \rightarrow K^{\mp} \pi^{ \pm}$decays}

One of the most suitable channels for charm mixing studies is $D^{0} \rightarrow K^{-} \pi^{+}$. The final state can be reached via two independent paths involving either no mixing and Cabibbo-favoured (CF) decays, or mixing and Doubly-Cabibbo suppressed (DCS) decays. Events of $D^{0} \rightarrow K^{-} \pi^{+}$are called right-sign (RS), and decays of $D^{0} \rightarrow K^{+} \pi^{-}$are referred to as wrong-sign (WS). The ratio of WS to RS events $R(t) \equiv \frac{N_{W S}(t)}{N_{R S}(t)} \approx R_{D}+\sqrt{R_{D}} y^{\prime} \frac{t}{\tau}+\frac{x^{\prime 2}+y^{\prime 2}}{4}\left(\frac{t}{\tau}\right)^{2}$ includes terms (in order of appearance) related to the the ratio of WS to RS non-mixed $D^{0}$ meson decays, the interference and the mixing of the charm mesons. Here, $t$ is the decay time, and $\tau$ is the measured $D^{0}$ lifetime. In this equation, the parameters $x^{\prime}$ and $y^{\prime}$ are rotated with respect to the charm mixing parameters $x$ and $y$ by a phase. The previous best measurement of the parameters $R_{D}, x^{\prime}$ and $y^{\prime}[10]$ is based on the charm prompt sample collected during LHCb Run 1 data taking period. Recently, a new DT data sample taken in LHCb Run 1 was analysed [11]. The new sample contains 1.7 million RS events, and 6.7 thousand WS events. When combining both samples, the DT events constitute only $2.5 \%$ of the full Run 1 LHCb statistics but the precision of the measured parameters improves by $10-20 \%$. The combined fit [11] tested three hypotheses: no $C P$ violation, no direct $C P$ violation and all $C P$ violation allowed. Under the last hypothesis, using the combined data sample, the results are:

- $R_{D}^{+}\left[10^{-3}\right]=3.474 \pm 0.081, \quad R_{D}^{-}\left[10^{-3}\right]=3.591 \pm 0.081$

- $\left(x^{\prime+}\right)^{2}\left[10^{-4}\right]=0.11 \pm 0.65, \quad\left(x^{\prime-}\right)^{2}\left[10^{-4}\right]=0.61 \pm 0.61$

- $\left(y^{+}\right)\left[10^{-3}\right]=5.97 \pm 1.25, \quad\left(y^{\prime-}\right)\left[10^{-3}\right]=4.50 \pm 1.21$

Here, the quantities are split by the flavour at production of the charm meson to allow for $C P$ violation detection. The quantities denoted with "+" refer to $D^{0}$ meson, and the ones with "-" refer to $\bar{D}^{0}$ meson. The results are compatible with no- $C P$ violation.

\subsection{Mixing and $C P$ violation in $D^{0} \rightarrow h^{0} \pi^{+} \pi^{-}$decays}

One way to independently measure the complex parameters $p, q$ and $\phi$, as well as the charm mixing parameters $x$ and $y$, is through a time-dependent Dalitz plot analysis, for instance through the decay $D^{0} \rightarrow h^{0} \pi^{+} \pi^{-}$. BaBar performed the first measurement of the $D^{0}-\bar{D}^{0}$ mixing parameters using a time-dependent amplitude analysis of the decay $D^{0} \rightarrow \pi^{0} \pi^{+} \pi^{-}$[12]. The experiment 
uses prompt $D^{0}$ decays, and the measured mixing parameters are $x=(1.5 \pm 1.2 \pm 0.6) \%$ and $y=(0.2 \pm 0.9 \pm 0.5) \%$.

For measurement of the charm mixing parameters, the LHCb analyses the so-called golden mode $D^{0} \rightarrow K_{\mathrm{s}}^{0} \pi^{+} \pi^{-}[13]$ employing a model-independent technique. The method uses as external input for the strong phase differences measured by the CLEO collaboration in pre-defined Dalitz bins [14]. The measured values by LHCb are $x=(-0.86 \pm 0.53 \pm 0.17) \%$ and $y=(0.03 \pm 0.46 \pm 0.13) \%$. The model-independent techniques are a very attractive possibility because the modelling of the Dalitz plot is associated with irreducible systematics due to the amplitude model. The uncertainty on the external measurements used in the model-independent techniques can be reduced further by using input from the experiment BESIII which is currently taking data, and a new measurement of the relative strong-phase difference parameters is underway, based on a sample four times bigger than the one collected by CLEO-c $[15,16]$. Moreover, this data sample is expected to grow by a factor of ten. The BESIII measurements are also fundamental for extracting the CKM angle $\gamma$ using the GGSZ method [17], and the uncertainty on $\gamma$ due to the external input can be reduced due to the larger BESIII statistics [18].

\section{Indirect $C P$ violation in charm}

The indirect $C P$ violation comprises the $C P$ violation in mixing, $A_{M}$, and interference between mixing and direct $C P$ violation, $A_{d}$, and it can be related to the observable $A_{\Gamma} \approx$ $\eta_{C P}\left[\frac{1}{2}\left(A_{M}+A_{d}\right) y \cos \phi+x \sin \phi\right] \equiv-a_{C P}^{i n d}$. The indirect $C P$ violation is accessible through measurement of the asymmetries between the effective lifetimes of charm decays to $C P$ eigenstates $A_{\Gamma}=\frac{\tau\left(\bar{D}^{0} \rightarrow h^{+} h^{-}\right)-\tau\left(D^{0} \rightarrow h^{+} h^{-}\right)}{\tau\left(\bar{D}^{0} \rightarrow h^{+} h^{-}\right)+\tau\left(D^{0} \rightarrow h^{+} h^{-}\right)}$. This type of $C P$ violation is universal, and measurements done with various final states e.g. $K^{+} K^{-}$and $\pi^{+} \pi^{-}$should be compatible. Within the Standard Model, this quantity is predicted to be smaller than $10^{-3}[19,20]$. A larger value than the predicted one, or a dependence of $A_{\Gamma}$ on the decay mode will indicate physics beyond the Standard Model. Measuring $A_{\Gamma}$ with CF decays $D^{0} \rightarrow K^{-} \pi^{+}$decays provides a useful null tests of the experimental technique - its value should be compatible with zero. Two independent analyses, using two different approaches were recently published by the LHCb collaboration [21]. Both measurements use the full LHCb Run 1 statistics of prompt charm decays to final states $K K$ and $\pi \pi$. One of the methods measures the asymmetries between the decay time in a complex two-stages unbinned fit involving the invariant masses $m\left(D^{0}\right), \Delta m\left(=m\left(D^{*+}\right)-m\left(D^{0}\right)\right)$, the $D^{0}$ decay time, and the impact parameter $\chi^{2}$ of the $D^{0}$ meson. The obtained results are: $A_{\Gamma}\left(K^{+} K^{-}\right)=(-0.14 \pm 0.37 \pm 0.10) \times 10^{-3}$ and $A_{\Gamma}\left(\pi^{+} \pi^{-}\right)=(0.14 \pm 0.63 \pm 0.15) \times 10^{-3}$. The largest source of systematic uncertainty for this method comes from modelling of the secondary backgrounds.

The other approach uses a binned approach. The reconstructed events data distributions are corrected for detector non-uniformities and presence of secondary decays, and the raw asymmetry is calculated in bins of $D^{0}$ decay time:

$$
A_{\mathrm{r} a w}=\frac{d N\left(D^{0}, t\right)-d N\left(\bar{D}^{0}, t\right)}{d N\left(D^{0}, t\right)+d N\left(\bar{D}^{0}, t\right)}
$$

The yields for calculating $A_{\text {raw }}$ are extracted by fitting the $\Delta m$ distribution. A linear fit of the $A_{\text {raw }}$ 
disribution versus the decay time is used for extracting $A_{\Gamma}$ :

$$
A_{\text {r } a w}=A_{0}-\frac{t}{\tau} A_{\Gamma}
$$

The linear slope of this distribution is not sensitive to direct $C P$ violation, nor to asymmetries due to production and detection asymmetries. The results of this method are $A_{\Gamma}\left(K^{+} K^{-}\right)=(-0.30 \pm$ $0.32 \pm 0.10) \times 10^{-3}$ and $A_{\Gamma}\left(\pi^{+} \pi^{-}\right)=(0.46 \pm 0.58 \pm 0.12) \times 10^{-3}$. The largest source of systematic uncertainty comes from the estimation of the secondary decays contribution. The results of the two independent approaches agree within one standard deviation. The results for $D^{0} \rightarrow K^{+} K^{-}$and $D^{0} \rightarrow \pi \pi$ are consistent, and show no evidence for $C P$ violation. These measurements improve on the precision of the previous best measurements [22] by nearly a factor of two.

A combination of the slightly more precise prompt measurement with the statistically independent secondary charm decays results based on the LHCb Run 1 [23] yields $A_{\Gamma}=(-0.29 \pm 0.28) \times$ $10^{-3}$. This is the most precise asymmetry measured in the charm system.

Belle recently reported their measurement using the final dataset of prompt charm decays $A_{\Gamma}=(-0.03 \pm 0.20 \pm 0.07) \times 10^{-2}[24]$.

The experiment also measured the related quantity, $y_{C P}=\frac{\tau\left(D^{0} \rightarrow K^{-} \pi^{+}\right)}{\tau\left(D^{0} \rightarrow h^{-} h^{+}\right)}$. In case of no-CP violation, $y_{C P}$ equals the $y$ charm mixing parameter. The Belle result using the same data set yields $y_{C P}=(1.11 \pm 0.22 \pm 0.09) \%$. Both observables are fitted simultaneously in bins of the cosine of the polar angle $\theta^{*}$ of the $D^{0}$ meson in centre-of-mass system with respect to the direction of $e^{+}$beam of the the KEKB asymmetric-energy $e^{+} e^{-}$collider [25].

The parameter $y_{C P}$ was measured by the BESIII experiment by taking advantage of quantum coherence between pairs of $D^{0}-\bar{D}^{0}$ mesons produced in $e^{+} e^{-}$annihilation near $\psi(3770)$ threshold. In this work, doubly-tagged events in which on $\mathrm{D}$ meson decays to a $C P$ eigenstate $\left(K^{+} K^{-}, \pi^{+} \pi^{-}, K_{\mathrm{S}}^{0} \pi^{0} \pi^{0}, K_{\mathrm{S}}^{0} \pi^{0}, K_{\mathrm{S}}^{0} \eta, K_{\mathrm{S}}^{0} \omega\right)$ and the other one to a semileptonic decays $(\mathrm{KeV}$, $K \mu \nu)$ are used. BESIII collaboration reports $y_{C P}=(-2.0 \pm 1.3 \pm 0.7) \%$ [26]. A new preliminary result was presented at this conference, based on events tagged with $C P$ tags $K_{\mathrm{L}}^{0} \pi^{0}$ and $K_{\mathrm{S}}^{0} \pi^{0}$ versus flavour tags $K e v, y_{C P}=(-0.98 \pm 2.43) \%$ [27].

\section{Direct CPV searches in two-body $D^{0} \rightarrow h^{+} h^{-}$charm decays}

The time-integrated $C P$ asymmetry in the decay rates of the singly Cabibbo-supressed process $D^{0} \rightarrow h^{+} h^{-}$

$$
A_{C P}\left(D^{0} \rightarrow h^{-} h^{+}\right) \equiv \frac{\Gamma\left(D^{0} \rightarrow h^{-} h^{+}\right)-\Gamma\left(\bar{D}^{0} \rightarrow h^{-} h^{+}\right)}{\Gamma\left(D^{0} \rightarrow h^{-} h^{+}\right)+\Gamma\left(\bar{D}^{0} \rightarrow h^{-} h^{+}\right)}
$$

was measured recently at $\mathrm{LHCb}$ [28]. This observable cannot be accessed directly due to the presence of production asymmetry of the $D^{0}$ and $\bar{D}^{0}$ mesons at $p p$ collider, and due to the presence of detection asymmetries. The latter can origin from an asymmetric interaction of the charged particles with the material of the detector. One example for this is the different cross-section for interaction with matter of $K^{+}$and $K^{-}$. Any asymmetry in the detector e.g. some inefficient regions, faulty electronics, etc could also contribute to the detection asymmetry. The effect of such asymmetries can cancel to first order with the regular swap of the magnetic field of the dipole magnet at LHCb. 
For prompt charm decays, the measured raw asymmetry in the number of background-subtracted number of signal decays

$$
A_{\text {raw }} \equiv \frac{N\left(D^{0} \rightarrow h^{+} h^{-}\right)-N\left(\bar{D}^{0} \rightarrow h^{+} h^{-}\right)}{N\left(D^{0} \rightarrow h^{+} h^{-}\right)+N\left(\bar{D}^{0} \rightarrow h^{+} h^{-}\right)}
$$

is related to the observable of interest, $A_{C P}\left(K^{-} K^{+}\right)$, via

$$
A_{C P}\left(D^{0} \rightarrow h^{-} h^{+}\right) \approx A_{\text {raw }}\left(D^{0} \rightarrow h^{-} h^{+}\right)-A_{P}\left(D^{*+}\right)-A_{D}\left(\pi^{+}\right) .
$$

This approximation is valid only for small asymmetries, this is why a particular care has been taken to exclude regions with large asymmetries, related to detector acceptance effects [28, 29].

To cancel the effect of the nuisance detection and production asymmetry, a set of Cabibbofavoured decays, $D^{0} \rightarrow K^{-} \pi^{+}, D^{+} \rightarrow K^{-} \pi^{+} \pi^{+}, D^{+} \rightarrow \bar{K}^{0} \pi^{+}$, in which no $C P$ violation is expected, is used. For example, by exploiting the prompt $D^{0} \rightarrow K^{-} \pi^{+}$channel, one can cancel the $D^{*+}$ production asymmetry, and the soft pion detection asymmetry. However, a new detection asymmetry is present due to the asymmetric final state $K^{-} \pi^{+}$. The effect of this asymmetry is mitigated using the other two calibration channels leading to

$$
\begin{aligned}
A_{C P}\left(D^{0} \rightarrow K^{-} K^{+}\right) & =A_{\text {raw }}\left(D^{0} \rightarrow K^{-} K^{+}\right)-A_{\text {raw }}\left(D^{0} \rightarrow K^{-} \pi^{+}\right) \\
& +A_{\text {raw }}\left(D^{+} \rightarrow K^{-} \pi^{+} \pi^{+}\right)-A_{\text {raw }}\left(D^{+} \rightarrow \bar{K}^{0} \pi^{+}\right) \\
& +A_{D}\left(\bar{K}^{0}\right)
\end{aligned}
$$

$A_{C P}\left(K^{-} K^{+}\right)$now only depends on measurable raw asymmetries and the calculable $\bar{K}^{0}$ detection asymmetry [30]. The method to determine $A_{C P}\left(K^{-} K^{+}\right)$has been first used in Ref. [31].

The time-integrated $C P$ asymmetry in $D^{0} \rightarrow K^{-} K^{+}$decays measured using the LHCb Run 1 prompt charm decays is determined to be

$$
A_{C P}^{\text {prompt }}\left(K^{-} K^{+}\right)=(0.14 \pm 0.15(\text { stat }) \pm 0.10(\text { syst })) \% .
$$

This result can be combined with previous LHCb measurements of the same and related observables. A combination with $A_{C P}\left(K^{-} K^{+}\right)$measured using secondary charm decays [31] yields

$$
A_{C P}^{\mathrm{comb}}\left(K^{-} K^{+}\right)=(0.04 \pm 0.12(\text { stat }) \pm 0.10(\text { syst })) \% .
$$

Since the same $D^{+}$decay channels are employed for the cancellation of detection asymmetries in secondary charm decays, the result is partially correlated with $A_{C P}^{\text {prompt }}\left(K^{-} K^{+}\right)$. The statistical correlation coefficient is $\rho_{\text {stat }}=0.36$. The difference in $C P$ asymmetries between $D^{0} \rightarrow K^{-} K^{+}$ and $D^{0} \rightarrow \pi^{-} \pi^{+}$decays, $\Delta A_{C P}^{\text {prompt }}$, is measured at LHCb using prompt charm decays [29]. A combination of the measurement of $A_{C P}^{\text {prompt }}\left(K^{-} K^{+}\right)$with $\Delta A_{C P}$ yields a value for

$$
\left.A_{C P}^{\text {prompt }}\left(\pi^{+} \pi^{-}\right)=A_{C P}^{\text {prompt }}\left(K^{+} K^{-}\right)-\Delta A_{C P}^{\text {prompt }}=(0.24 \pm 0.15 \text { (stat }) \pm 0.11 \text { (syst) }\right) \% .
$$

The statistical correlation coefficient of the two measurements is $\rho_{\text {stat }}=0.24$. The largest systematic uncertainties come from equalising the kinematic distributions for the final states $K K$ and $\pi \pi$, and from excluding regions with large nuisance asymmetries. 
A combination with the measurement using secondary charm results in

$$
A_{C P}^{\text {comb }}\left(\pi^{-} \pi^{+}\right)=(0.07 \pm 0.14(\text { stat }) \pm 0.11(\text { syst })) \%
$$

In conclusion, no evidence of $C P$ violation is found in the Cabibbo-suppressed decays $D^{0} \rightarrow$ $K^{-} K^{+}$and $D^{0} \rightarrow \pi^{-} \pi^{+}$. These results are obtained assuming that there is no $C P$ violation in $D^{0}-\bar{D}^{0}$ mixing and no direct $C P$ violation in the Cabibbo-favoured $D^{0} \rightarrow K^{-} \pi^{+}, D^{+} \rightarrow K^{-} \pi^{+} \pi^{+}$and $D^{+} \rightarrow \bar{K}^{0} \pi^{+}$decay modes. The combined LHCb results are the most precise measurements of the individual time-integrated $C P$ asymmetries $A_{C P}\left(K^{-} K^{+}\right)$and $A_{C P}\left(\pi^{-} \pi^{+}\right)$from a single experiment to date.

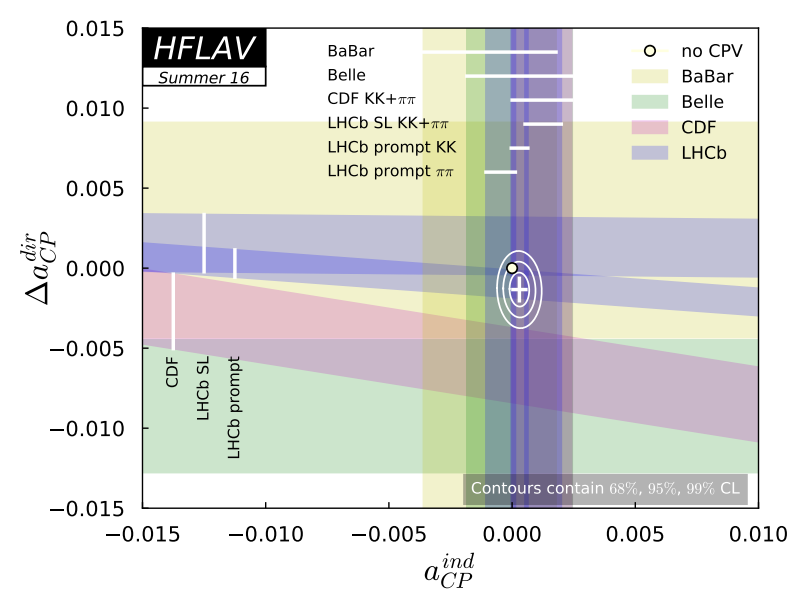

Figure 1: The combination plot shows the measurements for $\Delta A_{C P}$ and $A_{\Gamma}$ and sumarises the $C P$ violation searches in $D^{0} \rightarrow h^{+} h^{-}$decays using input from the BaBar, Belle, CDF and LHCb experiments.

Heavy Flavor Averaging Group [32] provides the combinations of all the independent searches for $C P$ violation in $D^{0} \rightarrow h^{+} h^{-}$decays. This is presented in Fig. 1.The vertical axis corresponds to the direct $C P$ violation, $\Delta a_{C P}^{\text {dir }}$, related to the observable $\Delta A_{C P} \equiv A_{C P}\left(K^{+} K^{-}\right)-A_{C P}\left(\pi^{+} \pi^{-}\right) \approx$ $\Delta a_{C P}^{\mathrm{d} i r}\left(1+y_{C P} \frac{\overline{\langle t\rangle}}{\tau}\right)+a_{C P}^{\mathrm{i} n d} \frac{\Delta\langle t\rangle}{\tau}$, where $\overline{\langle t\rangle}$ is the arithmetic average of the mean decay times of the $D^{0} \rightarrow K^{+} K^{-}$and $D^{0} \rightarrow \pi^{+} \pi^{-}$decays, and $\Delta\langle t\rangle$ is their difference. The indirect $C P$ violation asymmetry $a_{C P}^{\mathrm{ind}} \approx-A_{\Gamma}$ is displayed on the horizontal axis. The results of the global fit yield $a_{C P}^{\mathrm{ind}}=(0.030 \pm 0.026) \%$ and $\Delta a_{C P}^{\mathrm{dir}}=(-0.134 \pm 0.070) \%$. They are consistent with the no- $C P$ violation hypothesis in the charm sector at $9.3 \% \mathrm{CL}$.

\section{Direct CPV searches in other two- or three-body charm decays (phase-space integrated)}

Other singly Cabibbo-suppressed decays of the charm mesons can be used for complementary $C P$ violation searches as well. The ones with significant branching fractions are expected to yield more precise measurements. A search for $C P$ violation in $D^{+} \rightarrow \eta^{\prime} \pi^{+}$and $D_{s}^{+} \rightarrow \eta^{\prime} \pi^{+}$decays is conducted for a first time at a hadron collider using the full LHCb Run 1 data statistics. The $\eta^{\prime}$ meson is reconstructed via the final state $\pi^{+} \pi^{-} \gamma$. The signal sample contains about $63000 D^{ \pm}$ 
and $152000 D_{s}^{ \pm}$candidates. The $C P$ asymmetries are extracted with the aid of the control channels $D^{+} \rightarrow K_{\mathrm{S}}^{0} \pi^{+}$, with $K_{\mathrm{S}}^{0} \rightarrow \pi^{+} \pi^{-}$, and $D_{s}^{+} \rightarrow \phi \pi^{+}$, with $\phi \rightarrow K^{+} K^{-}$. The control channels are used to correct for production and detection asymmetries, in a similar way to the measurement done with $D^{0} \rightarrow h^{+} h^{-}$decays.

The measured $C P$-violating charge asymmetries are $A_{C P}\left(D^{+} \rightarrow \eta^{\prime} \pi^{+}\right)=(-0.61 \pm 0.72 \pm$ $0.53 \pm 0.12) \%$ and $A_{C P}\left(D_{s}^{+} \rightarrow \eta^{\prime} \pi^{+}\right)=(-0.82 \pm 0.36 \pm 0.22 \pm 0.27) \%$ [33], where the last uncertainty comes from external inputs related to $C P$ asymmetries measurements in the control channels. The results are the most precise measurement to date of these variables, and are consistent with $C P$ conservation. Prior to this, $D^{ \pm} \rightarrow \eta^{\prime} \pi^{ \pm}$and $D_{s}^{ \pm} \rightarrow \eta^{\prime} \pi^{ \pm}$decays have so far been studies only at $e^{+} e^{-}$colliders because of the experimental challenges in reconstructing $\eta^{(')}$ mesons in hadron collisions.

According to [34], the decays of $D^{0} \rightarrow K_{\mathrm{S}}^{0} K_{\mathrm{S}}^{0}$ are very sensitive to large beyond the Standard Model $C P$ violation effects. An upper limit for Standard Model $C P$ asymmetry is set to be below $1.1 \%$. The new measurement by Belle, $A_{C P}=(-0.02 \pm 1.53 \pm 0.17) \%$ [35] is compatible with $C P$ conservation. And so is the previous, less precise, measurement done by LHCb with Run 1 data, $A_{C P}=(2.9 \pm 5.2 \pm 2.2) \%[36]$.

The search of $C P$ violation in radiative charm decays $D^{0} \rightarrow V \gamma$, where $V$ stands for one of the light vector mesons $\phi, K^{*}, \rho$, is done for the first time by the Belle collaboration [37]. The search is motivated by the possibility to have sizeable $C P$ asymmetries in SM extensions with chromomagnetic dipole operators $[38,39]$. The search for $C P$ violation in radiative charm decays is complementary to the searches that have been exclusively performed in hadronic or leptonic decays. The reported results are: $A_{C P}\left(D^{0} \rightarrow \phi \gamma\right)=(-9.4 \pm 6.6 \pm 0.1) \%, A_{C P}\left(D^{0} \rightarrow K^{*} \gamma\right)=$ $(-0.3 \pm 2.0 \pm 0.0) \%, A_{C P}\left(D^{0} \rightarrow \rho \gamma\right)=(5.6 \pm 15.2 \pm 0.6) \%$

The BESIII collaboration measured the $C P$ asymmetry in semileptonic charm decays, $D^{+} \rightarrow$ $K_{\mathrm{L}}^{0} e v_{e}$. The result $A_{C P}\left(D^{+}\right)=(-0.59 \pm 0.60 \pm 1.48) \%$ was found to be compatible with the predicted value of $C P$ violation in the $K^{0}$ system, $-3.3 \times 10^{-3}$ [40].

The BESIII collaboration presented the preliminary result for $A_{C P}$ in four new modes, $A_{C P}\left(D^{+} \rightarrow\right.$ $\left.K_{\mathrm{S}}^{0} K^{+}\right)=(-1.5 \pm 2.8 \pm 1.6) \%, A_{C P}\left(D^{+} \rightarrow K_{\mathrm{S}}^{0} K^{+} \pi^{0}\right)=(1.4 \pm 4.0 \pm 2.4) \%, A_{C P}\left(D^{+} \rightarrow K_{\mathrm{L}}^{0} K^{+}\right)=$ $(-3.0 \pm 3.2 \pm 1.2) \%, A_{C P}\left(D^{+} \rightarrow K_{\mathrm{L}}^{0} K^{+} \pi^{0}\right)=(-0.9 \pm 4.1 \pm 1.6) \%$ [27], and found the results compatible with $C P$ asymmetry.

Most of the presented results in this section still have large experimental uncertainties, at the order of percent, which leaves room for new physics effects to manifest in future measurements.

\section{Direct CPV searches in multi-body charm decays $\left(D^{0} \rightarrow h^{+} h^{-} \pi^{-} \pi^{+}\right)$}

Multibody processes have rich decay dynamics which lead to large interference, and which can vary widely as a function of the phase space. They offer possibility to look for $C P$ violation in regions of phase space. Two main types of techniques are employed for these searches: model-dependent, and model-independent ones.

The model-independent techniques look for asymmetries in regions of phase space by counting. These kind of methods neither identify which amplitudes contribute to the observed asymmetry nor measure the actual asymmetry. There are two types of model-independent techniques-binned and unbinned. The binned analyses have to make an optimisation between minimising the number of 
bins, in order to maximise the number of events per bin, while retaining sensitivity to the interference between all contributing resonances without diluting the $C P$ asymmetries. A binned technique, using a $\chi^{2}$ difference method is introduced in [41], and used in [42-44]. The unbinned methods are based on the average weighted distance between events in phase space. Two unbinned methods have been used so far for searches of $C P$ violation of charm decays - nearest-neighbour method introduced in $[45,46]$ and used in [44], or the energy test first introduced in $[47,48]$, and its possible greater sensitivity for $C P$ violation searches was presented in [49].

The first application of the energy test in particle physics was the search for $C P$ violation in the decay channel $D^{0} \rightarrow \pi^{+} \pi^{-} \pi^{0}$ using LHCb data [50]. The first application for $C P$ violation in four-body decays was done with $D^{0} \rightarrow \pi^{+} \pi^{-} \pi^{-} \pi^{+}$decays using the full LHCb Run 1 prompt charm data sample [51]. The energy test employs a test statistic $T$ which is used to compare the average distances based on the metric function $\psi$. It is defined as

$$
T=\sum_{i, j>i}^{n} \frac{\psi_{i j}}{n(n-1)}+\sum_{i, j>i}^{\bar{n}} \frac{\psi_{i j}}{\bar{n}(\bar{n}-1)}-\sum_{i, j}^{n, \bar{n}} \frac{\psi_{i j}}{n \bar{n}},
$$

and the metric function $\psi_{i j} \equiv \psi\left(d_{i j}\right)=e^{-d_{i j}^{2} / 2 \sigma^{2}}$ is chosen as a Gaussian function with a tunable parameter $\sigma$ as it should be a falling function with increasing the distance between events. $T$ compares the average distances of pairs of events belonging to two samples of opposite flavour. The normalisation factor removes the impact of global asymmetries. The distance between two points in phase space is given by $d_{i j}=\left(m_{12}^{2, j}-m_{12}^{2, i}, m_{23}^{2, j}-m_{23}^{2, i}, m_{13}^{2, j}-m_{13}^{2, i}\right)$, where the $1,2,3$ subscripts indicate the final-state particles. For no-CPV, $T$ is expected to be zero, and larger than zero in case of $C P$ violation. This unbinned technique calculates a $p$-value under the hypothesis of $C P$ symmetry by comparing the nominal $T$ value observed in data to a distribution of $T$ values obtained from permutation samples, where the flavour of the $D^{0}$ is randomly reassigned to simulate samples without $C P$ violation. The $p$-value for the no $C P$ violation hypothesis is obtained as the fraction of permutation $T$ values greater than the nominal $T$ value. For four-body decay, the P-even and P-odd CP asymmetries can be tested. The P-even test is performed through the comparison of the distribution of events in the $D^{0}$ and $\bar{D}^{0}$ phase spaces, characterised by squared invariant masses. Additionally, characterising the events using a triple product of final-state particle momenta, $p\left(\pi_{i}\right)$, gives sensitivity to P-odd amplitudes, and thus allows the first test for P-odd $C P$ asymmetries in an unbinned model-independent technique. The parity-sensitive variable $C_{T}=p\left(\pi_{1}\right) \cdot\left(p\left(\pi_{2}\right) \times p\left(\pi_{3}\right)\right)$ is introduced, and the different flavours are analysed in regions with different sign of $C_{T}$.

The data are found to be consistent with the hypothesis of $C P$ symmetry with a p-value of $(4.6 \pm 0.5) \%$ in the P-even case, and marginally consistent with a p-value of $(0.6 \pm 0.2) \%$ in the P-odd case, corresponding to a significance for $C P$ non-conservation of 2.7 standard deviations. The deviation is located in a small phase-space region dominated by the $\rho^{0}$ resonance. The sensitivity of this method was studied using simplified simulation based on the model presented in [52] by introducing different types of $C P$ violation.

To really pin down the origin of the $C P$ violation, one should employ a model dependent technique in order to understand the nature of the interfering processes in a multibody decay. This means one has to develop a model of the intermediate particle resonances occurring in the decay. This amplitude model is used to describe the phase-space structure of the multibody final state. 
The resonant substructure of $\left(D^{0} \rightarrow \pi^{+} \pi^{-} \pi^{-} \pi^{+}\right)$is studied using data collected with the CLEO-c detector. At CLEO-c, the charm mesons are produced in quantum-correlated pairs $D^{0} \bar{D}^{0}$ at the resonance $\psi(3770)$. The flavour of the neutral charm mesons is tagged by looking the charge of a kaon associated with the decay of the other $D$ meson in the event e.g. the semileptonic decays $D^{0} \rightarrow K^{-} e^{+} v$. The analysis was carried on a CLEO-c legacy data set. About 7000 flavourtagged $\left(D^{0} \rightarrow \pi^{+} \pi^{-} \pi^{-} \pi^{+}\right)$events were used for an amplitude analysis, performed in order to disentangle the various intermediate state contributions. The most significant contributions are $D^{0} \rightarrow a_{1}(1260)^{+} \pi^{-}, D^{0} \rightarrow \sigma f_{0}(1370)$ and $D^{0} \rightarrow \rho(770)^{0} \rho(770)^{0}$. Using the information about the flavour tag of the $D^{0}$, a search for $C P$ violation is performed. The amplitude coefficients are allowed to differ between $D^{0}$ and $\bar{D}^{0}$ decays. The sensitivity varies between $4 \%$ and $22 \%$ for the different decay modes. No significant $C P$ violation is observed for any of the amplitudes. The global decay rate asymmetries between $D^{0}$ and $\bar{D}^{0}$ are measured to be $A_{C P}=\frac{N_{D^{0}}-N_{D^{0}}}{N_{D^{0}}+N_{D^{0}}}=(+0.54 \pm 1.04 \pm 0.51) \%$ [52].

Following a similar strategy, and data from the experiments CLEO II.V, CLEO III and CLEO-c was used to analyse the decays $\left(D^{0} \rightarrow K^{+} K^{-} \pi^{-} \pi^{+}\right)$. The flavour of the charm mesons collected with the CLEO II.V, CLEO III detectors is tagged by the charge of the pion in the strong decay $D^{*+} \rightarrow$ $D^{0} \pi^{+}$. About 3000 candidates were collected with the different CLEO experiment configurations. The dominant contributions are found to be $D^{0} \rightarrow \phi(1020) \rho(770)^{0}, D^{0} \rightarrow K_{1}(1270)^{+} K^{-}$and $D^{0} \rightarrow K(1400)^{+} K^{-}$. The result for the global asymmetry $A_{C P}=\frac{N_{D^{0}}-N_{D^{0}}}{N_{D^{0}}+N_{D^{0}}}=(+1.84 \pm 1.74 \pm 0.30) \%$ is consistent with $C P$ conservation [52]. .

\section{Conclusions and prospects}

The latest precision measurements in the charm sector at LHCb, BELLE, BESIII, BaBar, CLEO-c legacy data are presented in these proceedings.

$C P$ violation in the charm sector remains unobserved. All searches are consistent with no direct or indirect $C P$ violation, with limits approaching the order of $10^{-3}$ in the direct $C P$ violation searches, and $10^{-4}$ for indirect $C P$ violation searches. The no-mixing hypothesis for neutral charm mesons is excluded with a significance $>10$ standard deviations already with LHCb Run I prompt data, and the different results agree well. However, the charm mixing parameters remain to be measured with sufficient precision, and the input from the BESIII experiment is critical for these precision measurements.The key measurements are still statistically limited, and for future measurement the systematics is expected to reduce with statistics. Several key LHCb Run 1 analyses are still ongoing and several LHCb Run 2 analyses are now underway. A factor two gain in statistics has already been seen with LHCb Run 2 data due to trigger optimisation, and even more statistics is expected to be collected with the upgraded LHCb experiment.

With LHCb and BESIII continuing to take data, and the Belle II experiment joining soon, there are exciting times for the charm physics ahead.

\section{References}

[1] SLAC-SP-017, J. E. Augustin et al., Discovery of a Narrow Resonance in $e^{+} e^{-}$Annihilation, Phys. Rev. Lett. 33 (1974) 1406, [Adv. Exp. Phys.5,141(1976)].

[2] LHCb collaboration, R. Aaij et al., Observation of $D^{0}-\bar{D}^{0}$ oscillations, Phys. Rev. Lett. 110 (2013) 101802, arXiv:1211.1230. 
[3] M. Kobayashi and T. Maskawa, CP Violation in the Renormalizable Theory of Weak Interaction, Prog. Theor. Phys. 49 (1973) 652.

[4] A. Abashian et al., The Belle Detector, Nucl. Instrum. Meth. A479 (2002) 117.

[5] BaBar, B. Aubert et al., The BaBar detector, Nucl. Instrum. Meth. A479 (2002) 1, arXiv:hep-ex/0105044.

[6] CLEO, R. A. Briere et al., CLEO-c and CESR-c: A New frontier of weak and strong interactions, CLNS-01-1742 (2001).

[7] LHCb, A. A. Alves, Jr. et al., The LHCb Detector at the LHC, JINST 3 (2008) S08005.

[8] LHCb collaboration, R. Aaij et al., LHCb detector performance, Int. J. Mod. Phys. A30 (2015) 1530022, arXiv:1412.6352.

[9] BESIII, M. Ablikim et al., Design and Construction of the BESIII Detector, Nucl. Instrum. Meth. A614 (2010) 345, arXiv:0911.4960.

[10] LHCb collaboration, R. Aaij et al., Measurement of $D^{0}-\bar{D}^{0}$ mixing parameters and search for CP violation using $D^{0} \rightarrow K^{+} \pi^{-}$decays, Phys. Rev. Lett. 111 (2013) 251801, arXiv: 1309.6534.

[11] LHCb collaboration, R. Aaij et al., Measurements of charm mixing and CP violation using $D^{0} \rightarrow K^{ \pm} \pi^{\mp}$ decays, Phys. Rev. D95 (2017) 052004, arXiv:1611.06143, Erratum in preparation.

[12] BaBar, J. P. Lees et al., Measurement of the neutral D meson mixing parameters in a time-dependent amplitude analysis of the $D^{0} \rightarrow \pi^{+} \pi^{-} \pi^{0}$ decay, Phys. Rev. D93 (2016), no. 11 112014 , arXiv:1604.00857.

[13] LHCb collaboration, R. Aaij et al., Model-independent measurement of mixing parameters in $D^{0} \rightarrow K_{\mathrm{S}}^{0} \pi^{+} \pi^{-}$decays, JHEP 04 (2016) 033, arXiv:1510.01664.

[14] CLEO, J. Libby et al., Model-independent determination of the strong-phase difference between $D^{0}$ and $\bar{D}^{0} \rightarrow K_{S, L}^{0} h^{+} h^{-}(h=\pi, K)$ and its impact on the measurement of the CKM angle $\gamma / \phi_{3}$, Phys. Rev. D82 (2010) 112006, arXiv:1010.2817.

[15] R. A. Briere, Quantum coherent measurements, PoS CHARM2016 (2017) 031.

[16] BESIII, M.-G. Zhao, CP Violation, Mixing and nonleptonic decays at BESIII, PoS CHARM2016 (2017) 023.

[17] A. Giri, Y. Grossman, A. Soffer, and J. Zupan, Determining gamma using $B+-\longrightarrow$ DK+- with multibody D decays, Phys. Rev. D68 (2003) 054018, arXiv: hep-ph/0303187.

[18] LHCb, L. collaboration, Synergy of BESIII and LHCb physics programmes, LHCb-PUB-2016-025.

[19] M. Bobrowski, A. Lenz, J. Riedl, and J. Rohrwild, How Large Can the SM Contribution to CP Violation in $D^{0}-\bar{D}^{0}$ Mixing Be?, JHEP 03 (2010) 009, arXiv: 1002.4794.

[20] Y. Grossman, A. L. Kagan, and Y. Nir, New physics and CP violation in singly Cabibbo suppressed D decays, Phys. Rev. D75 (2007) 036008, arXiv: hep-ph/ 0609178.

[21] LHCb collaboration, R. Aaij et al., Measurement of the CP violation parameter $A_{\Gamma}$ in $D^{0} \rightarrow K^{+} K^{-}$and $D^{0} \rightarrow \pi^{+} \pi^{-}$decays, Phys. Rev. Lett. 118 (2017) 261803, arXiv:1702.06490.

[22] LHCb collaboration, R. Aaij et al., Measurements of indirect CP asymmetries in $D^{0} \rightarrow K^{-} K^{+}$and $D^{0} \rightarrow \pi^{-} \pi^{+}$decays, Phys. Rev. Lett. 112 (2014) 041801, arXiv:1310. 7201.

[23] LHCb collaboration, R. Aaij et al., Measurement of indirect $C P$ asymmetries in $D^{0} \rightarrow K^{-} K^{+}$and $D^{0} \rightarrow \pi^{-} \pi^{+}$decays, JHEP 04 (2015) 043, arXiv:1501.06777. 
[24] Belle, M. Staric et al., Measurement of $D^{0}-\bar{D}^{0}$ mixing and search for $C P$ violation in $D^{0} \rightarrow K^{+} K^{-}, \pi^{+} \pi^{-}$decays with the full Belle data set, Phys. Lett. B753 (2016) 412 , arXiv:1509.08266.

[25] S. Kurokawa and E. Kikutani, Overview of the KEKB accelerators, Nucl. Instrum. Meth. A499 (2003) 1.

[26] BESIII, M. Ablikim et al., Measurement of $y_{C P}$ in $D^{0}-\bar{D}^{0}$ oscillation using quantum correlations in $e^{+} e^{-} \rightarrow D^{0} \bar{D}^{0}$ at $\sqrt{s}=3.773 \mathrm{GeV}$, Phys. Lett. B744 (2015) 339, arXiv : 1501.01378.

[27] BESIII, X. Z. on behalf of BESIII collaboration, Charm hadron decay at BESIII, Contribution to these proceedings, FPCP17, Prague.

[28] LHCb collaboration, R. Aaij et al., Measurement of CP asymmetry in $D^{0} \rightarrow K^{+} K^{-}$decays, Phys. Lett. B767 (2017) 177, arXiv:1610.09476.

[29] LHCb collaboration, R. Aaij et al., Measurement of the difference of time-integrated CP asymmetries in $D^{0} \rightarrow K^{-} K^{+}$and $D^{0} \rightarrow \pi^{-} \pi^{+}$decays, Phys. Rev. Lett. 116 (2016) 191601, arXiv: 1602.03160.

[30] LHCb collaboration, R. Aaij et al., Search for direct CP violation in $D^{0} \rightarrow h^{-} h^{+}$modes using semileptonic B decays, Phys. Lett. B723 (2013) 33, arXiv:1303. 2614.

[31] LHCb collaboration, R. Aaij et al., Measurement of CP asymmetry in $D^{0} \rightarrow K^{-} K^{+}$and $D^{0} \rightarrow \pi^{-} \pi^{+}$ decays, JHEP 07 (2014) 041, arXiv: 1405.2797.

[32] Y. Amhis et al., Averages of b-hadron, c-hadron, and $\tau$-lepton properties as of summer 2016, arXiv:1612.07233, updated results and plots available at http://www.slac.stanford.edu/xorg/hflav.

[33] LHCb collaboration, R. Aaij et al., Measurement of CP asymmetries in $D^{ \pm} \rightarrow \eta^{\prime} \pi^{ \pm}$and $D_{s}^{ \pm} \rightarrow \eta^{\prime} \pi^{ \pm}$ decays, Phys. Lett. B771 (2017) 21, arXiv: 1701.01871.

[34] U. Nierste and S. Schacht, $C P$ Violation in $D^{0} \rightarrow K_{S} K_{S}$, Phys. Rev. D92 (2015), no. 5 054036, arXiv:1508.00074.

[35] A. Abdesselam et al., Measurement of CP asymmetry in the $D^{0} \rightarrow K_{\mathrm{S}}^{0} K_{\mathrm{S}}^{0}$ decay at Belle, arXiv:1609.06393.

[36] LHCb collaboration, R. Aaij et al., Measurement of the time-integrated CP asymmetry in $D^{0} \rightarrow K_{\mathrm{S}}^{0} K_{\mathrm{S}}^{0}$ decays, JHEP 10 (2015) 055, arXiv:1508.06087.

[37] Belle, A. Abdesselam et al., Observation of $D^{0} \rightarrow \rho^{0} \gamma$ and search for CP violation in radiative charm decays, Phys. Rev. Lett. 118 (2017), no. 5 051801, arXiv: 1603.03257.

[38] J. Lyon and R. Zwicky, Anomalously large $\mathscr{O}_{8}$ and long-distance chirality from $A_{\mathrm{CP}}\left[D^{0} \rightarrow\left(\rho^{0}, \omega\right) \gamma\right](t)$, arXiv:1210.6546.

[39] G. Isidori and J. F. Kamenik, Shedding light on CP violation in the charm system via D to V gamma decays, Phys. Rev. Lett. 109 (2012) 171801, arXiv:1205.3164.

[40] Z.-Z. Xing, Effect of KO - anti-KO mixing on CP asymmetries in weak decays of D and B mesons, Phys. Lett. B353 (1995) 313, arXiv: hep-ph/9505272, [Erratum: Phys. Lett.B363,266(1995)].

[41] I. Bediaga et al., On a CP anisotropy measurement in the Dalitz plot, Phys. Rev. D80 (2009) 096006, arXiv:0905.4233.

[42] LHCb collaboration, R. Aaij et al., Model-independent search for CP violation in $D^{0} \rightarrow K^{-} K^{+} \pi^{+} \pi^{-}$ and $D^{0} \rightarrow \pi^{-} \pi^{+} \pi^{-} \pi^{+}$decays, Phys. Lett. B726 (2013) 623, arXiv:1308.3189. 
[43] LHCb collaboration, R. Aaij et al., Search for CP violation in $D^{+} \rightarrow K^{-} K^{+} \pi^{+}$decays, Phys. Rev. D84 (2011) 112008, arXiv:1110.3970.

[44] LHCb collaboration, R. Aaij et al., Search for CP violation in the decay $D^{+} \rightarrow \pi^{-} \pi^{+} \pi^{+}$, Phys. Lett. B728 (2014) 585, arXiv:1310.7953.

[45] N. Henze, A multivariate two-sample test based on the number of nearest neighbor type coincidences, Ann. Statist. 16 (1988) 772.

[46] M. F. Schilling, Multivariate two-sample tests based on nearest neighbors, Journal of the American Statistical Association 81 (1986), no. 395 799, arXiv:http://www.tandfonline.com/doi/pdf/10.1080/01621459.1986.10478337.

[47] B. Aslan and G. Zech, New test for the multivariate two-sample problem based on the concept of minimum energy, Journal of Statistical Computation and Simulation 75 (2005), no. 2 109, arXiv:http://dx.doi.org/10.1080/00949650410001661440.

[48] B. Aslan and G. Zech, Statistical energy as a tool for binning-free, multivariate goodness-of-fit tests, two-sample comparison and unfolding, Nuclear Instruments and Methods in Physics Research Section A: Accelerators, Spectrometers, Detectors and Associated Equipment 537 (2005), no. 3 626 .

[49] M. Williams, Observing CP Violation in Many-Body Decays, Phys. Rev. D84 (2011) 054015, arXiv:1105.5338.

[50] LHCb collaboration, R. Aaij et al., Search for CP violation in $D^{0} \rightarrow \pi^{-} \pi^{+} \pi^{0}$ decays with the energy test, Phys. Lett. B740 (2015) 158, arXiv: 1410.4170.

[51] LHCb collaboration, R. Aaij et al., Search for CP violation in the phase space of $D^{0} \rightarrow \pi^{+} \pi^{-} \pi^{+} \pi^{-}$ decays, Phys. Lett. B769 (2017) 345, arXiv:1612.03207.

[52] P. d'Argent et al., Amplitude Analyses of $D^{0} \rightarrow \pi^{+} \pi^{-} \pi^{+} \pi^{-}$and $D^{0} \rightarrow K^{+} K^{-} \pi^{+} \pi^{-}$Decays, JHEP 05 (2017) 143, arXiv:1703.08505. 